\title{
Comparison of different non-linear equations and corresponding unitarization schemes
}

\section{O.V. Selyugin*}

Bogoliubov Laboratory of Theoretical Physics, JINR, 141980, Dubna, Russia

E-mail: seluginetheor.jinr.ru

\section{J.-R. Cudell}

Institut de Physique, Bât. B5a, Université de Liège, Sart Tilman, B4000 Liège, Belgium

E-mail: JR.Cudell@ulg.ac.be

Different forms of non-linear equations which mimic parton saturation in the non-perturbative regime are examined. These equations lead to corresponding unitarization schemes in the impact parameter representation of the hadron scattering amplitude. It is shown how specific properties of the non-linear equations reflect different features of the diffraction processes.

DIFFRACTION 2006 - International Workshop on Diffraction in High-Energy Physics September 5-10 2006

Adamantas, Milos island, Greece

${ }^{*}$ Speaker. 


\section{Introduction}

Future experiments at the LHC will open a new page in the physics of hadron-hadron interactions. The new energy region promises many new physical phenomena. It is very likely that in this region the asymptotic properties of the hadron-hadron interaction will appear. These properties are connected with the first principles of the theory such as analyticity, unitarity and Lorenz invariance. In the momentum transfer representation, some asymptotic bounds were obtained many years ago. For example, the AKM (Auberson-Kinoshita-Martin) [1] theorem leads to possible oscillation at very small $t$ in the elastic differential cross sections. Such oscillations may have already been discovered in the UA4/2 experiment [2,3]. In the impact parameter representation, a more complicated picture appears. For example, the Froissart-Martin theorem based on the first principles establishes a bound on the growth of total cross sections [4] - they cannot grow faster than $\log ^{2}\left(s / s_{0}\right)$. However, the Regge behavior and the linear BFKL equation lead to a power growth of the total cross sections. Obviously, this conflict requires a non-linear regime at super-high energies. This regime may be connected with the saturation of the density of the partons in hadron and can be related to the saturation of the unitarity bound only in the impact parameter representation.

The usual non-linear equations, such as the BK equation [5], are connected with perturbative and non-perturbative infrared effects that lead to the saturation regime at large impact parameters. Hence, they are not directly related to the Black Disk Limit (BDL)-saturation which in most models is connected with small impact parameters. Also, the models based on the color dipole representation of the high-energy interaction [6] suppress, for the most part, the soft hadron interaction. This is the reason why these models describes the hard interactions well. On the other hand, the BDL suppresses in most cases the hard component. It leads to an increase in the role of the soft component at super-high energies.

Unitarity of the scattering matrix, $S S^{+}=1$, is connected with the properties of the scattering amplitude in the impact-parameter representation as the latter is equivalent at high energy to a decomposition into partial-wave amplitudes. At high energies the scattering amplitude in the impact-parameter representation can saturate the unitarity bound of the overlapping function $G(s, b)$ below some impact parameter $b_{i}$. One can impose the unitarity condition through various schemes. Two of them are based on an analytic implementation of unitarity: the first is the standard eikonal representation

$$
T(s, t) \sim i \int_{0}^{\infty} b d b J_{0}(b q)[1-\exp (-\chi(s, b)],
$$

with $t=-q^{2}$. The second corresponds to the $U$-matrix approach [7]:

$$
G(s, b)=U(s, b) /[1-i U(s, b)] .
$$

where $U(s, b)=i \chi(s, b)=T_{\text {born }}(s, b)$. In [8] it was shown that such a solution can lead to a nonstandard behaviour of the ratio $\sigma_{e l} / \sigma_{t o t} \rightarrow 1$, as $s \rightarrow \infty$.

These schemes lead to a decrease in the growth of $\sigma_{t o t}$, but they predict very different energy regions for the onset of saturation. We show also [9] that such a decrease heavily depends on the profile in impact parameter dependence of the Born amplitude. In particular, if one takes the eikonal phase in the factorized form, one usually supposes that despite the fact that the energy 
dependence of $h(s)$ can be a power of $h(s) \sim s^{\Delta}$, the total cross section will satisfy the Froissart bound $\sigma_{t o t} \leq a \log ^{2}(s)$. We find in fact that the energy dependence of the imaginary part of the amplitude and hence of the total cross section depends on the form of $f(b)$, i.e., on the $s$ and $t$ dependence of the slope of the elastic scattering amplitude. For example, a power dependence on the impact parameter will still violate the Froissart-Martin bound after eikonalization.

\subsection{Saturation and non-linear equations}

Different approaches of the saturation of the scattering amplitude try to connect it with the non-linear saturation processes which have been considered in a perturbative QCD context $[10,11]$. Such processes lead to an infinite set of coupled evolution equations in energy for the correlation functions of the multiple Wilson lines. In the approximation where the correlation functions for more than two Wilson lines factorize, the problem reduces to the non-linear Balitsky-Kovchegov (BK) equation [5].

It is unclear how to extend these results to the non-perturbative region, but one will probably obtain a similar equation. In fact we found simple differential equations that reproduce either the $U$-matrix or the eikonal representation.

Let us consider the saturation equation that has been used for many years in the various fields of physics - that is the logistic equation:

$$
\partial N / \partial y=d N(1-\lambda N)
$$

This equation has a standard solution in the form

$$
N=f(b) e^{d \lambda y} /\left[1+\lambda f(b) e^{d \lambda y}\right] .
$$

If, inspired by the BK results, we take $d=\alpha(0)-1$ with $\alpha(0)$ the intercept of the Pomeron and use the evolution variable $y=\log s$, with $\lambda=1$, Eq. (1.4) coincides with the standard $U$-matrix representation of the scattering amplitude obtained in the $U$-matrix formalism for $\mathfrak{I} U(s, b)=s^{\Delta} f(b)$. Hence, Eq. (1.4) extends the unitarized form of the scattering amplitude and is determined by the non-linear equation (1.3). We can see that the asymptotic regime $(y=\log (s) \rightarrow \infty)$ leads to a bound $1 / \lambda$ for the amplitude, and gives the standard BDL bound for $\lambda=1$. We can use the same procedure [12] to obtain other unitarized forms of the scattering amplitude.

For example, from the non-linear equation

$$
\partial N / \partial y=d(1-\lambda N) \log (1-\lambda N)
$$

we obtain a solution

$$
N=\frac{1}{\lambda}\left[1-e^{-s^{d} / f(b)}\right]
$$

The equation reproduces the extended eikonal (quasieikonal) form [13]. With $\lambda=1$, Eq. (1.6) coincides with the standard eikonal form of the scattering amplitude in the impact parameter representation.

We show in Fig. 1 and Fig. 2 the dependence of these non-linear equations on the size of the intercept of the Pomeron and on the energy $S=\sqrt{s}$ in the case $\lambda=1$. We see that the qualitative 

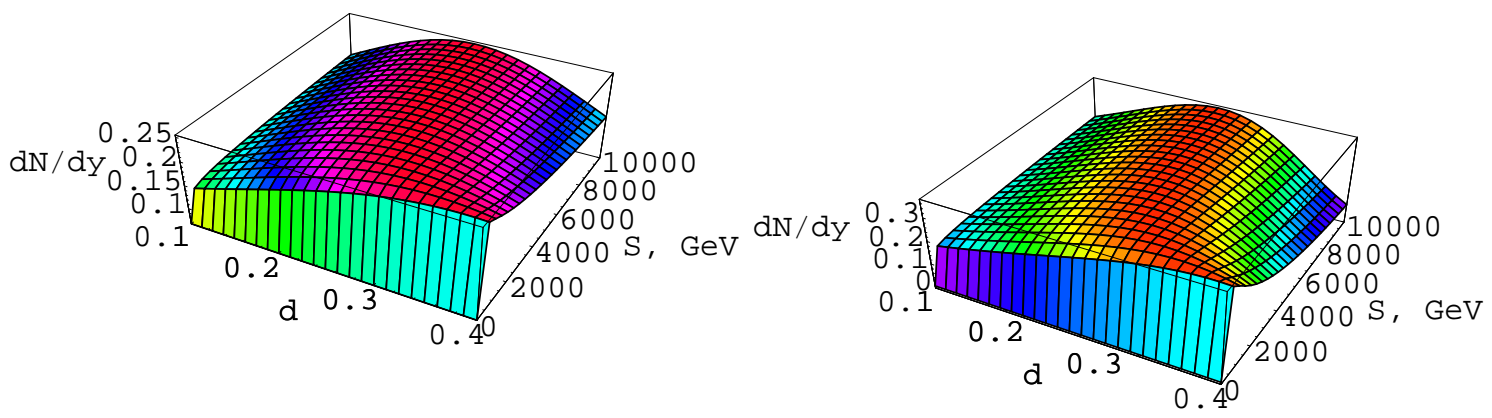

Figure 1: $d N / d y$ for Eq. (1.2) - left - and for Eq. (1.5) - right -.

behavior of both equations is very similar. The derivative in Eq. (1.6) has a larger maximum at low $s$ than that in Eq. (1.4). However, at large $s$, the situation in the opposite: the derivative in Eq. (1.4) exceeds that of Eq. (1.6).

We have shown that the most usual unitarization schemes could be recast into differential equations which are reminiscent of saturation equations [5]. Such an approach can be used to build new unitarization schemes and may also shed light on the physical processes underlying the saturation regime.

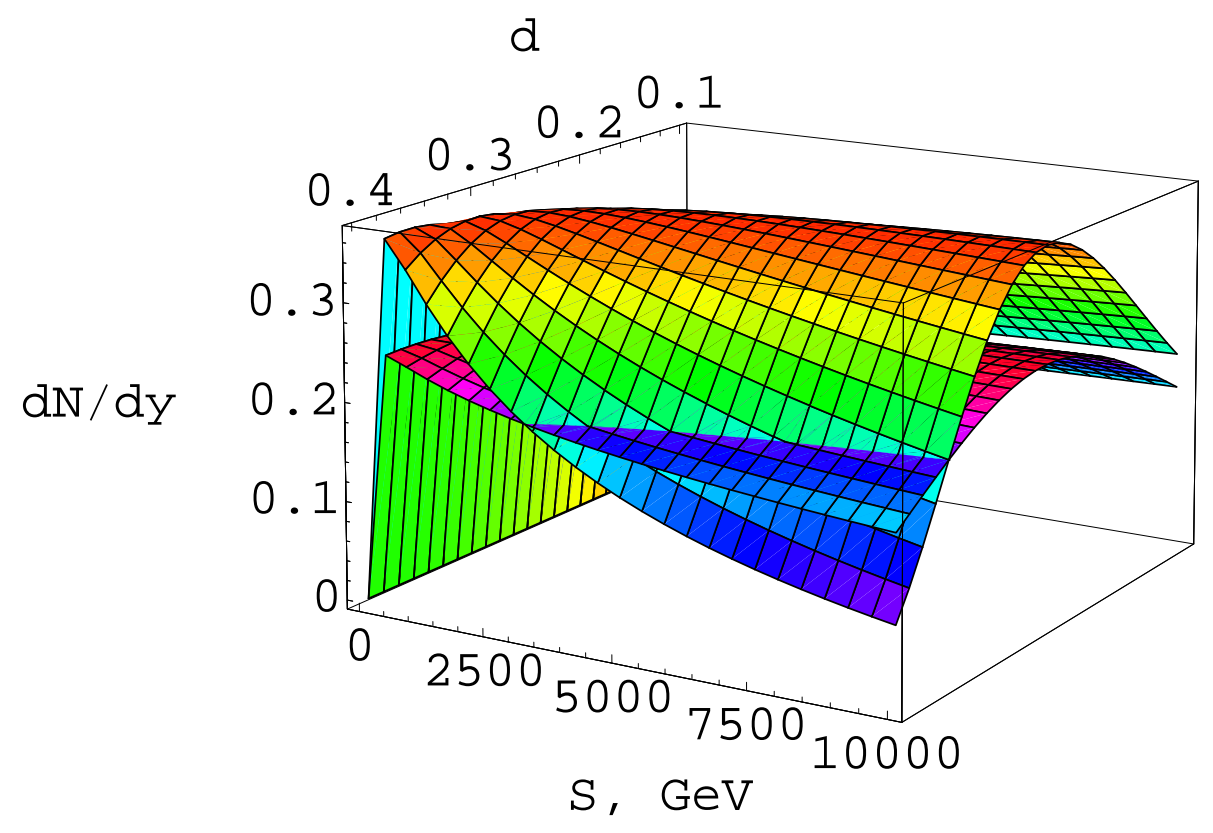

Figure 2: $d N / d y$ for Eq. (1.2) and for the Eq. ( 1.5) 
As an example of other unitarization schemes, the equation

$$
\frac{\partial N}{\partial y}=d N_{\text {bare }} / d t\left[1-N^{2}\right]
$$

has the unitarized solution

$$
G(s, b)=\tanh \left[N_{\text {bare }}\right]
$$

\subsection{Unitarization}

Let us now compare the behavior of the scattering amplitudes in different unitarization schemes. They are given by

$$
F(s, t)=i \int_{0}^{\infty} b d b G(s, b) J_{0}(q b)
$$

where $G(s, b)$ is the overlapping function which depends on the scheme and satisfies the BDL bound $G(s, b) \leq 1 . G(s, b)$ is a function of $s, b, N_{\text {bare }}$, and

$$
N_{\text {bare }}(s, b)=i \int_{0}^{\infty} q d q F_{\text {Born }}(s, q) J_{0}(q b) .
$$

If the Born scattering amplitude is in the Regge form

$$
F_{\text {Born }}(s, t)=i h s^{d} \exp \left(R^{2} / 2 t\right)
$$

the corresponding form in the impact parameter representation is given by

$$
F_{\text {Born }}(s, b)=i h \frac{s^{d}}{2 R^{2}} \exp \left(-b^{2} /\left(4 R^{2}\right)\right)
$$

This Born amplitude will be the input of the different unitarization schemes.

Following the Van Hove normalization [14], the corresponding total cross section is

$$
\sigma_{t o t}(s)=4 \pi \int_{0}^{\infty} b[G(s, b)] d b
$$

In Fig. 3, we show the total cross section for different unitarization schemes. For all the schemes, the Born amplitude was taken in the simplest Regge form (1.11) with the parameters corrsponding to the exchange of a hard Pomeron:

$$
h=0.1 ; \quad R=3 \mathrm{GeV}^{-1} ; \quad d=\alpha(0)-1=0.4
$$

Here we want to compare the schemes directly. It is clear that if we make a fit to experimental data, the constant $h$ can be different for different unitarization schemes.

We can see from Fig. 3 that at small energies $(\sqrt{s}=10 \mathrm{GeV})$ the total cross sections are practically the same for all unitarization schemes. At this energy the unitarized amplitude practically reduces to the Born amplitude. When the energy grows, the schemes differ most in an intermediate regime which corresponds to the energy region of approximately $30 \mathrm{GeV} \leq \sqrt{s} \leq 200 \mathrm{GeV}$. This regime corresponds to the growth of the overlapping function which is different in different 


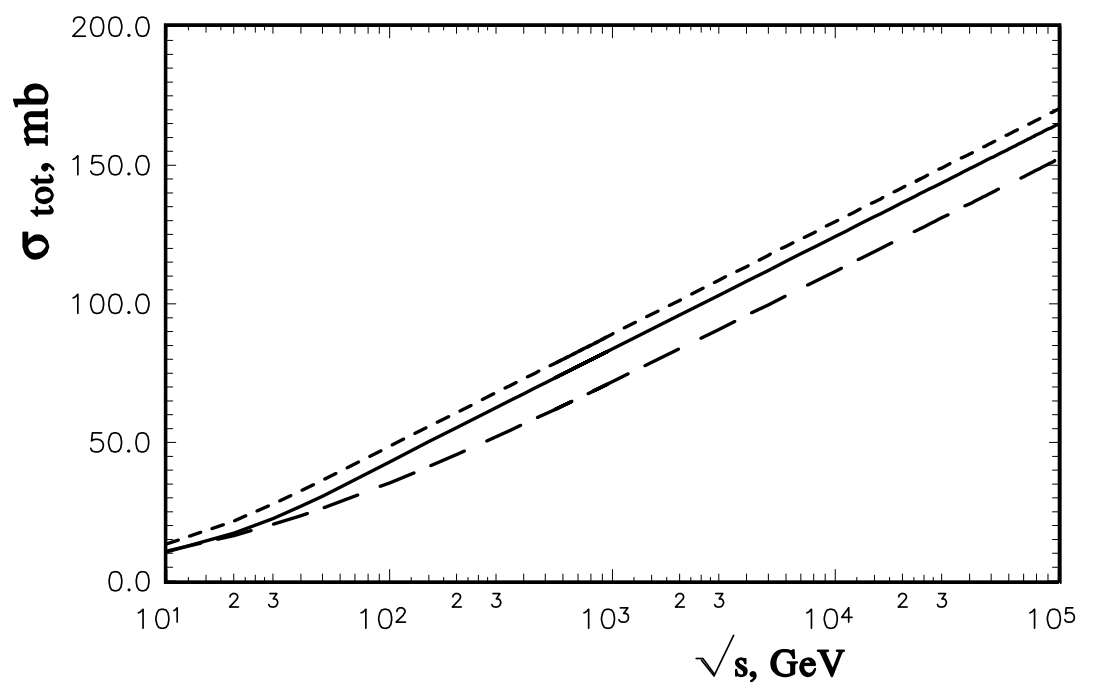

Figure 3: Total cross sections for different unitarization schemes. The hard, long-dashed and short-dashed lines correspond to the eikonal, the $U$-matrix and the tanh forms of unitarization.

unitarization schemes. However, when we come to the asymptotic regime (with saturation of the BDL bound) the difference ceases and the growth is practically the same in a wide energy region.

Hence, the asymptotics of all unitarization schemes are practically the same. Such a behavior leads to two methods of analysing experimental data. One is connected with a different contribution of the secondary Reggeons to the cross section. In this case, the asymptotics of the total cross sections can be exactly the same for different unitarization procedures. The second solution is obtained if we take the same contributions of the secondary Reggeons. The asymptotics of $\sigma_{\text {tot }}$ will then have the same energy dependence but some quantitative difference between the different unitarization schemes. In this case, the comparison of the predictions for the total cross sections at LHC energies from the different fitting procedures with different unitarization schemes will point out the true unitarization scheme and the true form of the non-linear equation.

\section{Conclusion}

In the presence of a hard Pomeron [15], the saturation effects can change the behavior of some features of the cross sections already at LHC energies. Hence, the problem of unitarization of the scattering amplitude will be very important for the analysis of experimental data.

Non-linear effects which work in the whole energy region supply an acceptable growth of the total cross sections. Saturation leads to a relative growth of the contribution of peripheral interactions. The most usual unitarization schemes can be recast into differential equations which are reminiscent of saturation equations. We can see that the different forms of the non-linear 
equations lead to the different behavior of the scattering amplitudes at intermediate energies and to the same behavior at asymptotic energies. Further exploration of the unitarization schemes and of their connection with the future experimental data of LHC may shed some light on the physical processes underlying the saturation regime at the partonic level.

\section{Acknowledgments}

O.V.S. thanks the Chairmen of the Organized Committee of Conference C. Ktorides and R. Fiore for the invitation and financial support. O.V.S. acknowledges the support of FRNS (Belgium) for visits to the University of Liège where part of this work was done. We thank E. Martynov, S. Lengyel, G. Soyez and P.V. Landshoff for discussion.

\section{References}

[1] G. Auberson, T. Kinoshita and A. Martin, Phys. Rev. D 3 (1971) 3185.

[2] UA4/2 Coll., C. Augier et al., Phys. Lett. B 316, (1993) 448.

[3] P. Gauron, B. Nicolescu and O.V. Selyugin, Phys. Lett. B 397 (1997) 305.

[4] M. Froissart, Phys. Rev. 123 (1961) 1053; A. Martin, Nuov. Cim. A 42 (1965) 930.

[5] J. Balitsky, Nucl. Phys. B 463 (1996) 99; Y.V. Kovchegov, Phys. Rev. D 60 (1999) 0340008 ; Phys. Rev. D 61 (2000) 074018.

[6] A.H. Mueller, Nucl. Phys. B 415 (1994) 373.; ibid. B 437 (1995) 107.

[7] A.A. Logunov, V.I. Savrin, N.E. Tyurin, and O.A. Khrustalev, Theor. Mat. Fiz. 6 (1971) 157.

[8] S.M. Troshin and N.E. Tyurin, Phys. Lett. B 316 (1993) 316.

[9] J.-R. Cudell, O.V. Selyugin, Czech. J. Phys. 55 (2005) A235.

[10] L.V. Gribov, E. Levin and M. Ryskin, Phys. Rep. 49 (1994); A. Mueller and J.W. Qiu, Nucl. Phys. B 286 (1986) 427.

[11] L. McLerran and R. Venugopalan, Phys. Rev. D 49 (1994) 2233; ibid. 3352.

[12] J.-R. Cudell, O.V. Selyugin, Nucl. Phys. B (Proc. Suppl.) 146 (2005) 185 [hep-ph/0412338].

[13] K.A. Ter-Martirosyan, Sov. ZhETF Pisma 15 (1972) 519; A.B. Kaidalov, L.A. Ponamarev, K.A. Ter-Martirosyan, Sov. J. Part. Nucl. 44 (1986) 468.

[14] L. Van Hove, Rev. Mod. Phys. 36 (1964) 655.

[15] J.-R. Cudell, A. Lengyel, E. Martynov and O.V. Selyugin, Phys. Lett. B 587 (2004) 78; Nucl.Phys. A 755 (2006) 587; [hep-ph/0501288]. 